\title{
新型弯曲形双偶氮苯类液晶合成及光致异构性能研究
}

\author{
刘曼曼 \\ 刘豪浩跒道仁 \\ 张智勇* \\ 关金涛 \\ (武汉轻工大学化学与环境工程学院 武汉 430023)
}

\begin{abstract}
摘要 弯曲形偶氮苯液晶由于其偶氮键独特的光致可逆异构化性能, 已成为光电子信息材料研究的热门课题, 但光响 应速度慢已成为制约其发展的关键因素. 目前报道的弯曲型偶氮类液晶化合物的偶氮键都距离中心核较远的位置，光 致异构的响应时间较长, 大都在分钟以上, 不利于光敏器件应用研究. 本工作试图以 2-甲基-1,3-间苯二胺为中心核，将 偶氮键紧挨中心核两边，末端为直链烷基，设计合成了一系列新型弯曲形双偶氮苯类液晶化合物，以期缩短光响应时 间. 通过 IR, ${ }^{1} \mathrm{H} \mathrm{NMR},{ }^{13} \mathrm{C}$ NMR 和 ICP-MS 光谱鉴定这些化合物的分子结构, 经差示扫描量热仪(DSC)和偏光显微镜 (POM)测定其液晶相变温度和相态织构; 并通过测定 2-甲基-1,3-双(4-((4-庚基苯基)酯基)-1-(E)-偶氮苯基)苯(2c)的紫 外-可见光的吸收光谱研究其光致异构化性能, 通过 UV-Vis 光谱仪和偏光显微镜(POM)测定其液晶化合物以及掺杂向 列相液晶材料的光致异构现象和响应时间. 实验结果表明, 所有设计合成的弯曲形双偶氮苯类液晶化合物均具有近晶 相相态，且相态温度范围较宽，当近晶相态化合物 $\mathbf{2 c}$ 掺杂到向列相混合液晶中时，其光致异构响应时间为 $2 \sim 3 \mathrm{~s} ，$ 在日 光下液晶态恢复时间为 $3 \sim 4 \mathrm{~s}$, 在乙酸乙酯稀溶液中时 $10 \mathrm{~s}$ 可达到光稳态. 说明这类弯曲形双偶氮液晶化合物具有较 快的光致异构响应速度.
\end{abstract}

关键词 偶氮苯; 弯曲形液晶; 光致异构; 合成

\section{Synthesis and Photo-Induced Isomerization Performance for the Novel Bent Diazobenzene Liquid Crystals}

\author{
Liu, Manman Liu, Haohao $\quad$ Yan, Daoren Zhang, Zhiyong* Guan, Jintao \\ (Department of Chemistry and Environmental Engineering, Wuhan Polytechnic University, Wuhan 430023)
}

\begin{abstract}
The photo-reversible isomerization property of the azo bend liquid crystal has become a hot topic in the research opt-electronic information materials, but its slow light response has become a key factor restricting its development. At present, the azo bond of the bent liquid crystal compound is far from the central nucleus, and the photo isomerization response time is long, mostly over minutes, which is not conducive to the application research of the photosensitive device. In this work, methyl-1,3-m-phenylenediamine was chosen the central nucleus. The azo bond is adjacent to both sides of the central ring and the terminal group is an alkyl chain. A series of novel curved bisazo benzene liquid crystal compounds were designed and synthesized, in order to shorten the light response time. The molecular structures of these compounds were identified by IR, ${ }^{1} \mathrm{H}$ NMR, ${ }^{13} \mathrm{C}$ NMR and ICP-MS spectroscopy. The phase transition temperature and phase texture of the liquid crystals were determined by differential scanning calorimeter (DSC) and polarized light microscopy (POM). The photo isomerization performance of 2-methyl-1,3-bis(4-((4-heptylphenyl)ester)-1-(E)-azophenyl)benzene (2c) was determined by UV-Vis absorption spectroscopy. The photo isomerism and response time of the liquid crystal compound and the doped nematic liquid crystal material were measured by UV-Vis spectrometer and polarizing microscope (POM). The experimental results show that all the curved bisazo benzene liquid crystal compounds designed and synthesized have the smectic phase and the phase temperature range is wide. The photo induced isomerization response time of compound $\mathbf{2 c}$ in the liquid crystal state of smectic phase and nematic phase is $2 \sim 3 \mathrm{~s}$, and the recovery time of liquid crystal state in sunlight is $3 \sim 4 \mathrm{~s}$, which can be reached in diluted ethyl acetate solution for $10 \mathrm{~s}$. This type of bent bisazo liquid crystal compound has a relatively fast photo induced heterogeneous response speed.
\end{abstract}

Keywords azobenzene; curved liquid crystal; photoisomerism; synthesis

* Corresponding author. E-mail: zzy6211@126.com

Received March 1, 2019; revised April 8, 2019; published online September 5, 2019.

Project supported by the National Nutural Science Foundation of China (Nos. 11074054, 11374067).

国家自然科学基金(No. 11074054, 11374067)资助项目. 
弯曲形偶氮苯类液晶化合物以其独特的光致可逆 异构化性能而倍受学术界广泛关注, 其在分子开关 ${ }^{[1]}$ 、 光信息储存 ${ }^{[2]}$ 、集成光学以及光控液晶分子取向等方面 具有潜在的应用研究和开发价值. 2008 年 Rahman 等 ${ }^{[3]}$ 首次报道了对偶氮苯液晶的光致异构性能研究，提出了 偶氮键在光控液晶分子取向上的应用, 引起了偶氮苯液 晶的研究热潮. 2012 年, Nagaveni 等 ${ }^{[4]}$ 合成了以间苯二 酚为中心核的偶氮弯曲形液晶, 虽然有液晶相态, 但没 有向列相, 且光致异构化速度在 $5 \mathrm{~min}$ 以上, 转化速率 较慢. 同年董寅 ${ }^{[5]}$ 设计合成了一系列弯曲形偶氮液晶化 合物, 探究了液晶分子中心核的弯曲度与紫外可见吸收 光谱的关系, 合成的偶氮液晶分子由反式转化为顺式的 异构化速度在 $1 \mathrm{~min}$ 左右, 而顺式到反式的热恢复弛豫 一般需要 $1 \mathrm{~h}$ 以上. 2014 年周瑛 ${ }^{[6]}$ 合成了含单/双偶氮基 团的丙烯酸酯类弯曲形液晶, 着重比较了两类弯曲形偶 氮液晶的光致异构性能, 其中双偶氮基团的弯曲形液晶 在紫外光照 $2 \mathrm{~min}$ 左右时光致异构转化达到平衡, 而单 偶氮液晶则需要 $20 \mathrm{~min}$, 可见偶氮键的数量会影响液晶 分子的光响应速率. 2013 年 Rahman 等 ${ }^{[7]}$ 合成的吡啶环 类弯曲形偶氮液晶在近晶相液晶态时 $9.4 \mathrm{~s}$ 达到光稳态. 2015 年 Rahman 等 ${ }^{[8]}$ 再次报道了以邻苯酚为中心核的双 偶氮弯曲形液晶, 其光致异构化速度在 $15 \mathrm{~s}$ 左右. 2017 年 Aralapura 等 ${ }^{[9]}$ 报道了由硫醇取代的偶氮苯衍生物与 金纳米材料核心通过 $\mathrm{Au}-\mathrm{S}$ 键键合而成的基于偶氮苯 的金纳米材料，光致异构至饱和态的时间约 24 s. 2017 年 $\mathrm{He}$ 等 ${ }^{[10]}$ 合成了一系列具有支链末端的手性偶氮苯化 合物, 并对其光敏性能进行了研究, 发现光化学性质受 偶氮苯的反一顺式构型及偶氮键与手性中心之间的连接 位置影响较大. 而现有报道 ${ }^{[7 \sim 15]}$ 的这些弯曲形液晶化合 物中, 偶氮基团作为桥键大都引入在离分子中心核较远 的侧臂环上, 光致异构响应时间较长, 都是分钟级别以 上, 不利于进行基础光电性质及其应用研究, 且近几年 在该领域的研究报道较少.

因此，研究开发光致异构时间短、光响应速度快、 宽液晶相态温度范围的新型弯曲形偶氮苯类液晶化合
物成为发展关键. 本工作试图以 2-甲基-1,3-间苯环为中 心核，将双偶氮键引入到中心核附近作为桥键，烷基链 为末端基团, 设计合成了一系列新型偶氮弯曲形液晶化 合物 $\mathbf{2} \mathbf{a} \sim 2 \mathrm{~g}$, 以期获得具有较快光致异构化速度的弯 曲形液晶化合物. 通过差示扫描量热仪测定目标化合物 各个相态的温度区间. 再通过投射偏光熔点仪观察每个 化合物在各个温度区间内的相态结构, 由此确定每个化 合物在不同温度区间内的相态种类. 同时通过紫外-可 见吸收光谱(UV-Vis)对其光致异构性能进行了研究.

\section{1 结果与讨论}

\section{1 目标化合物 $2 a \sim 2 g$ 的合成}

化合物 $\mathbf{2 a} \sim \mathbf{2 g}$ 的合成路线如 Scheme 1 所示, 所合 成的 7 个新型目标化合物经过 IR, ${ }^{1} \mathrm{H}$ NMR, ${ }^{13} \mathrm{C} \mathrm{NMR}$ 和 MS 对其结构进行表征, 结果表明这些化合物的分子结 构正确.

\section{2 目标化合物 $2 a \sim 2 g$ 的液晶相态分析}

目标化合物 $\mathbf{2 a} \sim \mathbf{2 g}$ 的液晶相转变温度通过差示扫 描量热法(DSC)测定，其测试数据见表 1 和图 1. 由表 1 和图 1 可知, 化合物 $\mathbf{2 a} \sim \mathbf{2 g}$ 均为近晶相液晶相态, 随着 末端柔性链的长度增加 $(n<10)$, 熔点与清亮点都有所 下降, 且液晶相态温度范围随着末端碳链增长而加宽. 这可能是由于随着烷基链增长 $(n<10)$, 分子柔性增加,

表 1 目标化合物 $\mathbf{2 a} \sim \mathbf{2 g}$ 的相变温度 ${ }^{a}$

Table 1 Phase transition temperatures of the target compounds $\mathbf{2 a} \sim 2 \mathrm{~g}$

\begin{tabular}{cccc}
\hline Compd. $n$ & Heating $/{ }^{\circ} \mathrm{C}\left[\Delta H /\left(\mathrm{kJ} \bullet \mathrm{mol}^{-1}\right)\right]$ & $\Delta T /{ }^{\circ} \mathrm{C}$ \\
\hline 2a & 5 & Cr 119.36(20.22) Sm 150.22 (12.27) Iso & 30.86 \\
2b & 6 & Cr 112.32 (24.39) Sm 150.64 (7.09) Iso & 38.32 \\
2c & 7 & Cr 102.58 (30.32) Sm 144.32 (6.38) Iso & 41.74 \\
2d & 8 & Cr 92.13 (29.8) Sm 138.67 (9.50) Iso & 46.54 \\
2e & 9 & Cr 72.36(24.74) Sm 136.26 (10.94) Iso & 63.9 \\
2f & 10 & Cr 70.20(29.04)Sm 135.32 (8.72) Iso & 65.12 \\
2g & 12 & Cr 81.67 (26.46) SmE 138.54 (13.97) Iso & 56.87 \\
\hline
\end{tabular}

${ }^{a} \mathrm{Cr}$ represents the crystalline phase, $\mathrm{Sm}$ represents the smectic phase, $\mathrm{N}$ represents the nematic phase; Iso represents the isotropic liquid.

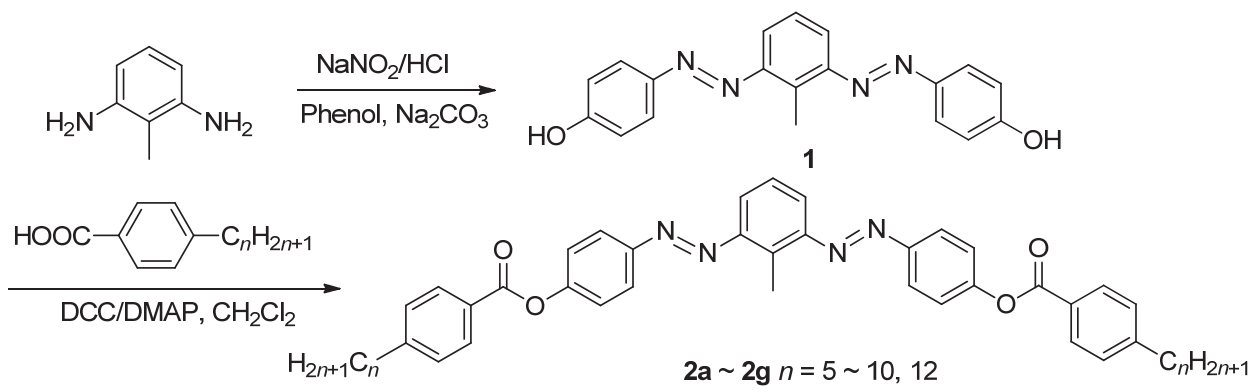

图式 1 目标化合物 $\mathbf{2 a} \sim \mathbf{2 g}$ 的合成路线

Scheme 1 Synthetic routes of target compounds $2 \mathrm{a} \sim 2 \mathrm{~g}$ 


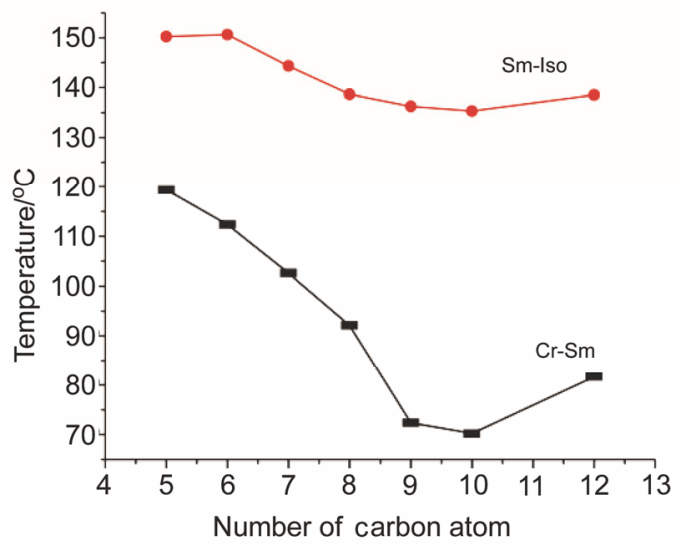

图 1 目标化合物 $\mathbf{2 a} \sim \mathbf{2 g}$ 的相变温度曲线图

Figure 1 Phase transition temperature graph of target compounds $\mathbf{2 a} \sim \mathbf{2 g}$

减小了分子之间相互作用力，使得熔点降低. 当末端烷 基链过长 $(n>10)$ 时, 其化合物的熔点因分子量增加而 升高, 而当熔点过高时, 很难进行液晶性能研究.

\section{3 化合物 $2 \mathrm{~g}$ 的相态结构分析}

根据目标化合物 $\mathbf{2 g}$ 的 DSC 曲线(图 2a)，在升温过程 中, 当加热到 $81.67{ }^{\circ} \mathrm{C}$ 时, 出现了一个强吸热峰, 热焓

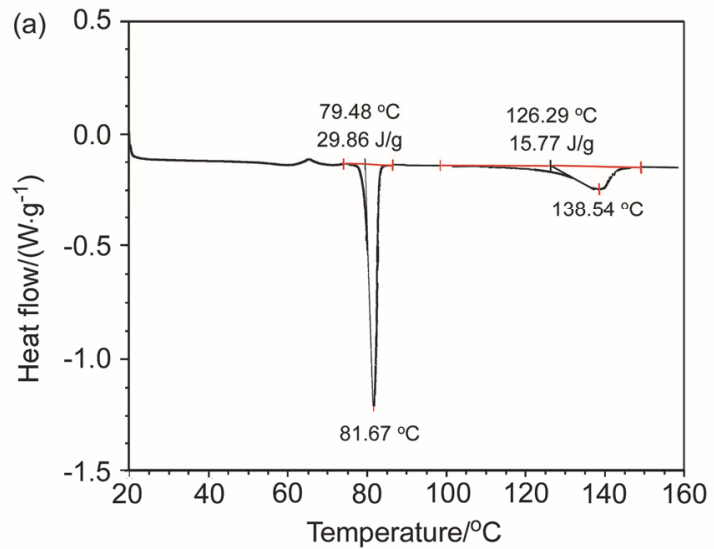

(b)

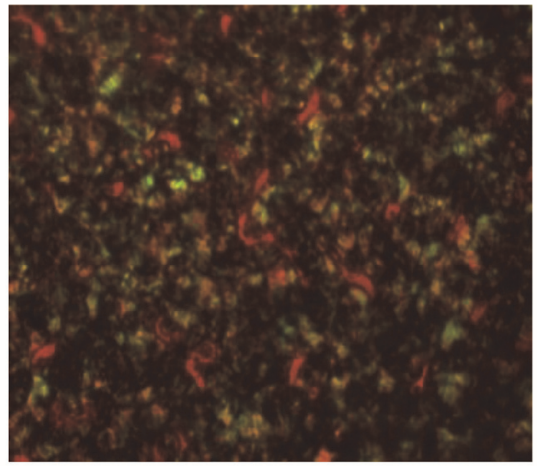

图 2 (a)目标化合物 $\mathbf{2 g}$ 的 DSC 曲线和(b)在 $100{ }^{\circ} \mathrm{C}$ 时的近晶 相 $\mathrm{E}$ 相态织构图

Figure 2 (a) DSC curve and (b) smectic E textures at $100{ }^{\circ} \mathrm{C}$ of compound $2 \mathrm{~g}$
变化较大, 这是典型的晶体相态 $(\mathrm{Cr})$ 转变为近晶相态 $(\mathrm{Sm})$ 的吸收峰, 即为熔点. 当加热到 $138.54{ }^{\circ} \mathrm{C}$ 时, 近晶 相转变为各向同性液体，即清亮点为 $138.54{ }^{\circ} \mathrm{C}$; 说明 该化合物近晶相温度范围较宽, 达到了 $56.87{ }^{\circ} \mathrm{C}$. 图 $2 \mathrm{~b}$ 是目标化合物 $2 \mathrm{~g}$ 降温过程中 $100{ }^{\circ} \mathrm{C}$ 时在偏光显微镜下 观察到的相态织构图, 是一种典型的近晶相镶嵌织构 图.

\section{4 化合物 $2 \mathrm{c}$ 在溶剂中的 UV 光致异构性能}

图 3 是目标化合物 $\mathbf{2 c}$ 在 $365 \mathrm{~nm}$ 紫外光照射下发生 反一顺异构化谱图. 由图 3 可知, 波长位于 $330 \sim 340 \mathrm{~nm}$ 处有强吸收峰，位于 $430 \sim 450 \mathrm{~nm}$ 处有弱吸收峰，这是 由偶氮键反式的 $\pi-\pi^{*}$ 电子跃迁和顺式的 $n-\pi *$ 电子跃迁 所引起的吸收. 在 $365 \mathrm{~nm}$ 紫外光照射下，随着时间的延 长, 目标化合物 $2 \mathrm{c}$ 在 $443 \mathrm{~nm}$ 处特征吸收峰增强, 而在 $345 \mathrm{~nm}$ 处特征吸收峰减弱, 表明溶剂体系中的反式结 构通过光照后不断减少, 顺式结构不断增加, 而且顺式 结构没有反式结构稳定，因而发生了顺一反异构. 此外 随着光照时间的延长, 在 $443 \mathrm{~nm}$ 左右处的特征吸收峰 和 $345 \mathrm{~nm}$ 左右处的特征吸收峰均出现了不同程度的蓝 移，说明 $\mathbf{2 c}$ 中的酯基抑制了电子的共振效应，使分子不 能较好地处于平面共轭结构.

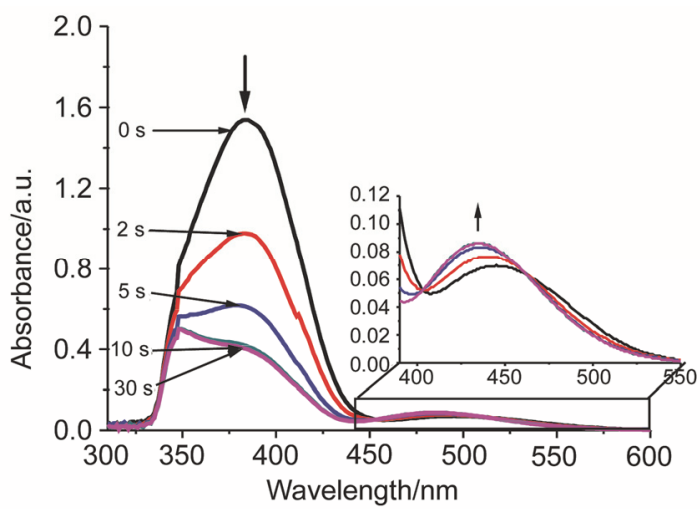

图 3 化合物 $2 \mathrm{c}$ 的 UV 光谱图 $\left(1 \times 10^{-5} \mathrm{~mol} / \mathrm{L}\right.$ 石油醚溶液, 365 $\mathrm{nm}, 1 \mathrm{~mW} / \mathrm{cm}^{2}$ )

Figure 3 Ultraviolet-absorption spectra of compound $2 \mathrm{c}$ in UV $\left(1 \times 10^{-5} \mathrm{~mol} / \mathrm{L}\right.$ petroleum ether solution, $\left.365 \mathrm{~nm}, 1 \mathrm{~mW} / \mathrm{cm}^{2}\right)$

由图 3 可知, 化合物 $\mathbf{2 c}$ 在紫外光照 $10 \mathrm{~s}$ 后达到光 稳态，化合物在光稳态下顺式异构体所占比例可根据式 (1) 计算 ${ }^{[14]}$ :

$$
f(\text { cis })=\frac{[\mathrm{cis}]_{\mathrm{t}}}{[\operatorname{trans}]_{0}}=\left(1-\frac{A_{t}}{A_{0}}\right) /\left(1-\varepsilon_{\text {cis }} / \varepsilon_{\text {trans }}\right)
$$

$A_{0}$ 为反式异构体在最大吸收波长处的初始吸光度值; $A_{\mathrm{t}}$ 为经紫外光饱和照射后同一波长处吸光度值; $\varepsilon_{c i s}$ 和 $\varepsilon_{\text {trans }}$ 分别为顺式和反式异构体的摩尔消光系数, 对于小分子 偶氮化合物而言 $\varepsilon_{c i s} / \varepsilon_{\text {trans }}=0.051^{[16]}$. 假定光照前化合物 
全部以反式结构存在，所合成的弯曲型双偶氮化合物 2c 的光转化率由上述公式计算后可达到 $77.95 \%$ (其中最 大吸收波长为 $334 \mathrm{~nm}, A_{0}$ 为 $1.537, A_{\mathrm{t}}$ 为 0.4 ). 相比结构 相似的偶氮苯棒状液晶化合物的 $11 \% \sim 55 \%$, 光转化率 有明显提高 ${ }^{[17]}$, 而通常偶氮苯小分子体系经饱和紫外 光照射后, 顺式异构体所占比例 $<80 \%{ }^{[18]}$, 可见目标液 晶化合物 $2 \mathrm{c}$ 的光转化率接近了小分子体系的最高光转 化率. 另外该系列其它化合物也具有同样的光致异构性 能特点, 例如化合物 $2 \mathrm{~d}$ 在与 $\mathbf{2 c}$ 相同的实验条件下, 紫 外光照 $10.1 \mathrm{~s}$ 可达到光稳态, 与化合物 $\mathbf{2 c}$ 相较之下仅慢 了 $0.1 \mathrm{~s}$.

\section{5 化合物 $2 c$ 及其掺加在向列相液晶中的光致异构性} 能研究

图 $4 \mathrm{a}$ 为化合物 $\mathbf{2 c}$ 在 UV 光没有照射时呈现的近晶 相 $\mathrm{E}$ 态织构图, 当 UV 光照 $3 \mathrm{~s}$ 后, 液晶相态消失, 转化
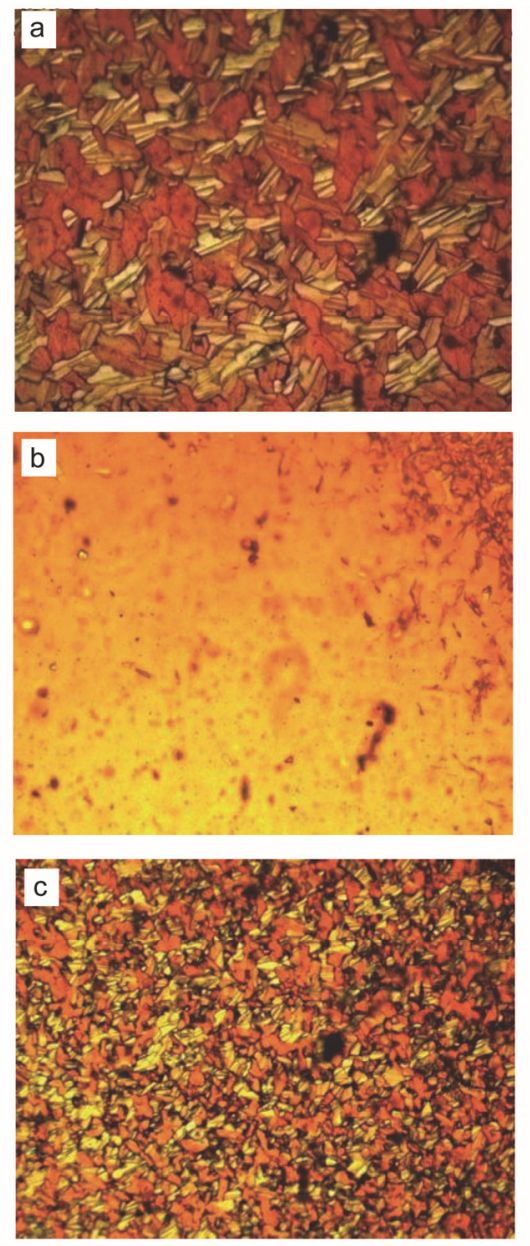

图 4 目标化合物 $\mathbf{2 c}$ 在 $120{ }^{\circ} \mathrm{C}$ 时 UV 光照导致近晶相态织构 变化的 POM 图

Figure 4 Smectic E texture changes of target compound $2 \mathrm{c}$ in POM at $120{ }^{\circ} \mathrm{C}$

(a) Before UV irradiation; (b) $3 \mathrm{~s}$ after UV irradiation; (c) $4 \mathrm{~s}$ after removing UV irradiation
为各向同性态(图 4b), 说明偶氮键由反式转化为顺式, 分子间的有序排列被破坏. 当撤走 UV 光，在自然光下 放置 $4 \mathrm{~s}$ 后, 偶氮液晶分子又恢复到近晶相态(图 4c), 说 明偶氮键由顺式转化为反式结构. 从图 4c 还可以看出, 尽管是近晶相态，但所呈现的相态织构畴区变得更加密 集，说明分子排列发生了改变，分子排列更加紧密. 该 系列其他化合物在相同实验条件下也显现出了相像的 顺反异构转化.

图 5 为母体液晶 HAG54800-200 与掺杂弯曲液晶化 合物 2c 的 DSC 谱图. 图 6 为掺杂后的混合液晶材料以 及未掺杂前的母体液晶在 UV 照射下与撤走 UV 光照后

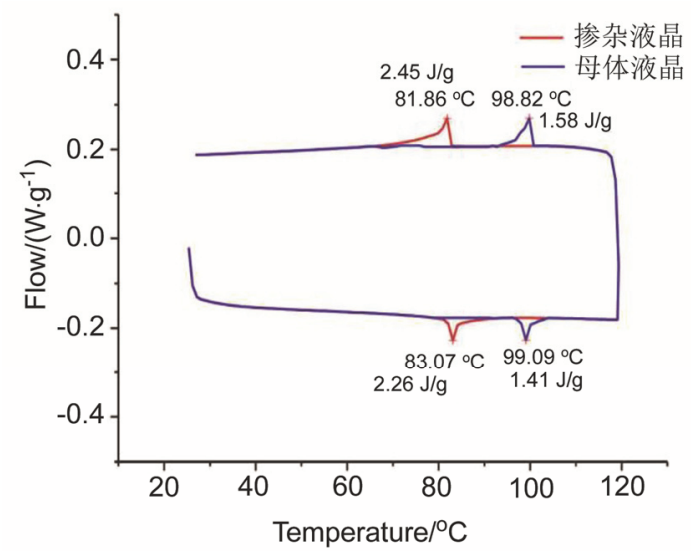

图 5 母体液晶 HAG54800-200 与弯曲液晶 $\mathbf{2 c}$ 掺杂后的 DSC 图

Figure 5 DSC diagram of parent liquid crystal HAG54800-200 and curved liquid crystal $2 \mathrm{c}$ doped
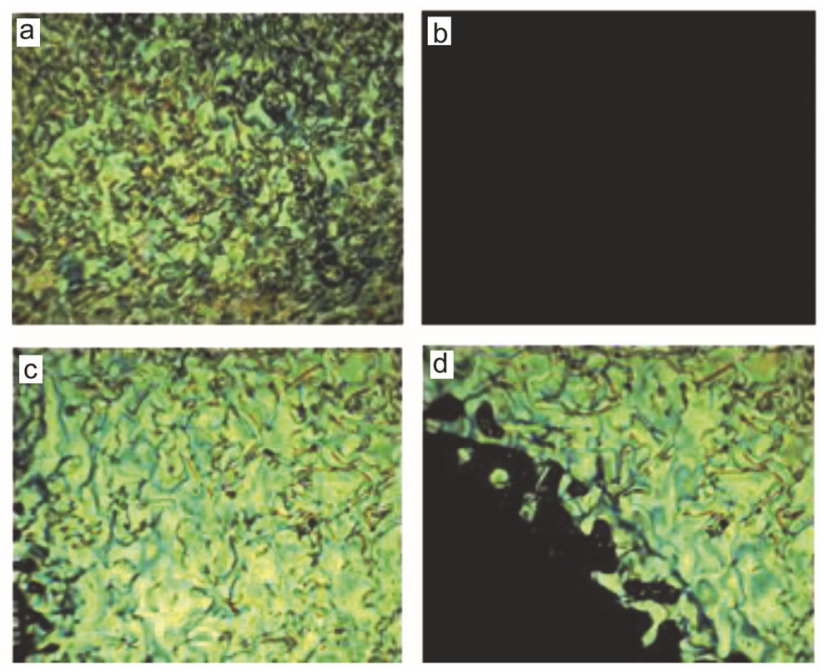

图 6 化合物 $2 \mathrm{c}$ 的混合液晶在 $60{ }^{\circ} \mathrm{C}$ 时 $\mathrm{UV}$ 光致异构向列相 态织构变化的 POM 图

Figure 6 POM changes of nematic phase texture for the LCs mixture with compound $\mathbf{2 c}$ in UV-light isomerization at $60{ }^{\circ} \mathrm{C}$

(a) Before UV irradiation; (b) $2 \mathrm{~s}$ after UV irradiation; (c) $3 \mathrm{~s}$ after removing UV irradiation; (d) bottom left conner: UV irradiation for $2 \mathrm{~s}$, upper right corner: no UV irradiation 
的液晶相态的变化情况. 掺杂化合物 $\mathbf{2 c}$ 后的混合液晶 材料相态发生变化, 而对于没有掺杂化合物 $2 \mathrm{c}$ 的向列 相液晶材料 HAG54800-200 无任何相态变化. 紫外光照 $2 \mathrm{~s}$ 后向列相态转化为各向同性态织构, UV 光照导致液 晶分子的反式偶氮键转化为顺式偶氮结构, 使液晶原本 的向列相态消失, 变成各向同性的液态. 当撤走 UV 光 $3 \mathrm{~s}$ 后, 混合液晶又重新恢复了向列相态织构. 用 UV 光 - 日光照射这个 $\mathbf{2 c}$ 掺杂的混合液晶反复光致异构实验 10 次, 响应速度无明显差别, 混合液晶相态温度的清亮 点也无明显变化, 说明化合物 $\mathbf{2 c}$ 掺杂到向列相混合液 晶材料中的光致稳定性良好. 将 $\mathbf{2 a}$ 和 $2 \mathrm{~g}$ 也分别掺杂到 液晶 HAG54800-200 中进行 UV-Vis 光致异构实验, 也得 到了同样的效果, 且用 $\mathbf{2 a}$ 掺杂的响应速度比 $\mathbf{2}$ 快 $0.2 \mathrm{~s}$.

所合成的目标化合物 $\mathbf{2 c}$ 在近晶相态和向列相态液 晶中都能发生光致异构现象, 在近晶相液晶态时的光响 应时间 $3 \mathrm{~s}\left(120{ }^{\circ} \mathrm{C}\right)$, 恢复时间 $4 \mathrm{~s}$ 左右; 将其与母体液 晶掺杂混合后形成的向列相态液晶材料, 其 UV 光致异 构响应时间达到 $2 \mathrm{~s}$ 左右, 其恢复时间 $3 \mathrm{~s}$ 左右 $\left(60{ }^{\circ} \mathrm{C}\right)$; 这与现有文献[7]报道的在固体中紫外光照时反式 $\rightarrow$ 顺 式为 $9.4 \mathrm{~s}$, 可见光照时顺式 $\rightarrow$ 反式为 $200 \mathrm{~min}$ 相比, 其 光响应速度有了很大的提升. 分析原因可能是化合物 2c 的双偶氮键结构在照射前是弯曲结构, 分子与分子 间联动性较大, 分子内的一个偶氮键转动, 会带动分子 间另一个偶氮键和其他分子偶氮键的转动, 从而增加了 光响应速度, 表明这类弯曲型双偶氮苯类液晶具有更快 的 UV 光响应功能. 同时, 实验中还发现掺杂目标化合 物 2c 的向列相混合液晶在低于 $45{ }^{\circ} \mathrm{C}$ 时不能发生光致 异构现象, 这可能是因为在此光照强度 $\left(1 \mathrm{~mW} / \mathrm{cm}^{2}\right)$ 下, 随着温度的降低, 液晶粘度或分子间作用力增大, 导致 UV 光能量不足以使偶氮键克服向列相液晶分子间的作 用力而发生顺一反异构现象.

\section{6 弯曲形双偶氮苯液晶 UV 光致异构机理推断}

利用网络 ChemBioDraw Ultra 12.0 软件模拟目标化 合物分子的 3D 结构图, 推测弯曲形双偶氮苯液晶分子 中的两个偶氮键基团, 在紫外(UV)光照射下液晶分子 发生反一顺异构翻转的顺序为: (trans,trans) $\rightarrow$ (trans, $c i s) \rightarrow(c i s, c i s)$. 反式偶氮苯结构团所占比例逐渐降低, 顺式偶氮苯基团所占比例逐渐升高, 液晶分子有序排列 逐渐破坏, 液晶态也就逐渐消基失. 当 UV 撤去以后, 在相同温度可见光作用下, 很快发生(cis, cis $) \rightarrow$ (trans, cis $) \rightarrow($ trans, trans) 逆转过程, 形成反式偶氮键结构, 分 子排列成有序状态, 恢复液晶相态(图 7). 在上述的顺反 异构过程中, 分子构形发生了巨大变化, 而这种分子构 形的变化导致分子整体排列产生变化, 偶氮键处于顺式 状态时液晶性质消失, 从而影响液晶态的光学性质 ${ }^{[2]}$.

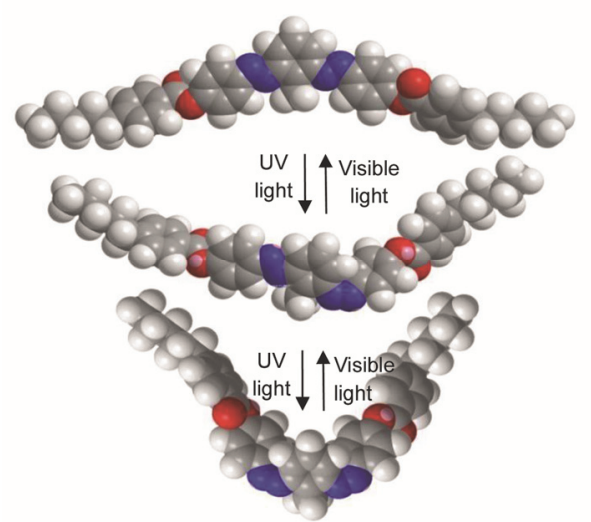

图 7 弯曲形双偶氮苯液晶分子的顺反异构转化 3D 示意图 Figure 7 3D-schematic diagram of the cis-trans isomerization for the bent diazobenzene liquid crystal molecules

\section{2 结论}

设计合成了 7 个新型结构的弯曲型双偶氮苯液晶化 合物 $\mathbf{2 a} \sim 2 \mathrm{~g}$, 所有目标化合物分子结构都通过 IR, ${ }^{1} \mathrm{H}$ NMR, ${ }^{13} \mathrm{C}$ NMR, ICP-MS 进行表征, 实验结果表明这些 目标化合物的分子结构正确; 通过差示扫描量热仪 (DSC)和偏光显微镜(POM) 对所有目标化合物进行液晶 相态测定, 结果表明这些弯曲形双偶氮苯类液晶化合物 2a $\sim 2 \mathrm{~g}$ 具有较宽的近晶液晶相态，最宽达到 $65.12{ }^{\circ} \mathrm{C}$ (化合物 2f), 而且液晶态温度范围随着末端碳链增长呈 现先增大后减小的趋势. 通过紫外光(UV)、可见光(Vis) 以及偏光显微镜 $(\mathrm{POM})$ 对目标化合物 $\mathbf{2 c}$ 进行光致异构 性能测试, 结果表明, 目标化合物 $\mathbf{2 c}$ 在浓度为 $1 \times 10^{-5}$ $\mathrm{mol} / \mathrm{L}$ 的乙酸乙酯稀溶液中具有良好的光致异构性能, UV 光照 $10 \mathrm{~s}$ 可达到光稳态, 光转化率能达到 77.95. 目 标化合物 $2 \mathrm{c}$ 经 UV 光照射 2 3 $\mathrm{s}$ 即改变相态并达到光 稳态, 光转化恢复时间为 $3 \sim 4 \mathrm{~s}$. 与母体向列相液晶材 料 HAG54800-200 混合配制的混合向列相液晶材料经 $\mathrm{UV}$ 光照 $2 \mathrm{~s}$ 发生光致异变, 液晶态消失, 当撤走 $U V$ 光 照 $3 \mathrm{~s}$ 又迅速恢复液晶态, 反复用 $U V$ 光 - 日光照射的光 致异构实验 10 次, 其响应速度无明显差别, 而且液晶相 态液晶态温度范围和清亮点也无明显变化, 说明化合物 2c 掺杂到向列相混合液晶材料中的光致稳定性良好, 同时表明这类新型弯曲型双偶氮苯类液晶化合物具有 较快的光致异构响应速度, 为今后进一步研究快速光响 应弯曲液晶材料提供了良好的实验素材.

\section{3 实验部分}

\section{1 仪器与试剂}

Thermo 公司 330 FT-IR 型红外光谱仪; Thermo 公司 LTQ XL 型质谱仪, ESI 离子源 $(35 \mathrm{eV})$, 气源为高纯度氮 气(纯度>99.999\%); Varian 公司 INOVO $400 \mathrm{MHz}$ 核磁 
共振仪(TMS 内标); 德国 Vario EL Cude 型元素分析仪; 北京泰克公司 X-4 型数字显微熔点仪; TA 公司 Q-10 型 差示扫描量热仪(DSC), 升温速率: $5{ }^{\circ} \mathrm{C} / \mathrm{min}$; XPN-100E 投射偏光熔点仪; 广州邦沃公司的 FUV-6BK 型点光源 型 UV-LED 光固化装置; 上海元析公司 UV-8000S 紫 外-可见(UV-Vis)分光光度计.

2-甲基-1,3-间苯二胺，国药集团化学试剂有限公司; 二环已基碳二亚胺(DCC), 南京化学试剂公司；4-二甲 氨基吡啶(DMAP), 山东西亚化学工业有限公司; 向列 相混合液晶 HAG54800-200, 江苏和成显示科技股份有 限公司. 4-正戊基苯甲酸、4-正己基苯甲酸、4-正庚基苯 甲酸、4-正辛基苯甲酸、4-正壬基苯甲酸、4-正癸基苯 甲酸和 4-正十二烷基苯甲酸均是本实验室合成.

\section{2 合成方法}

\subsubsection{2-甲基-1,3-双(4-羟基偶氮苯基)苯的合成}

向三口烧瓶中加入冷冻的浓盐酸 $40 \mathrm{~mL}(0.5 \mathrm{~mol})$, 控制瓶内温度低于 $-25{ }^{\circ} \mathrm{C}$, 在慢速摚拌下滴加由 12.5 $\mathrm{g}(0.18 \mathrm{~mol})$ 亚硝酸钠与 $18 \mathrm{~mL}$ 水配成的溶液, 加完后再 分多次慢慢加入由 2-甲基-1,3-间苯二胺 $(0.05 \mathrm{~mol})$ 与 25 $\mathrm{mL}$ 浓盐酸配成的悬浮液, 并逐渐加快搅拌速度. 滴加 完毕后, 继续反应 $0.5 \mathrm{~h}$, 得到黄色透明液体, 加入 $4.8 \mathrm{~g}$ $(0.08 \mathrm{~mol})$ 尿素, 搅拌至无气泡产生, 得重氮盐待用.

向另一三口瓶中加入 $11 \mathrm{~g}(0.12 \mathrm{~mol})$ 苯酚、 $31.8 \mathrm{~g}$ (0.3 mol) 碳酸钠和 $200 \mathrm{~mL}$ 水, 缓慢滴加所得重氮盐, 冷 水浴下反应 $3 \mathrm{~h}$, 薄层色谱(TLC)跟踪反应至反应完全, 抽滤, 滤饼用乙醇重结晶, 得 $11.32 \mathrm{~g}$ 2-甲基-1,3-双(4-羞弪 基偶氮苯基)苯(1). 黄色晶体, 收率 68.2\%. m.p. 145 $148{ }^{\circ} \mathrm{C} ;{ }^{1} \mathrm{H}$ NMR $\left(400 \mathrm{MHz}, \mathrm{CDCl}_{3}\right) \delta: 7.97(\mathrm{~d}, J=8 \mathrm{~Hz}$, $2 \mathrm{H}), 7.717(\mathrm{~d}, J=8 \mathrm{~Hz}, 4 \mathrm{H}), 7.28(\mathrm{t}, J=4 \mathrm{~Hz}, 4 \mathrm{H}), 5.13(\mathrm{~s}$, 2H), $2.11(\mathrm{~s}, 3 \mathrm{H}) ;{ }^{13} \mathrm{C}$ NMR $\left(100 \mathrm{MHz}, \mathrm{CDCl}_{3}\right) \delta: 159.51$, 151.84, 147.11, 129.65, 126.27, 125.29, 120.39, 115.60, 14.10; FT-IR (KBr) v: 3327.11, 2920.96, 2851.94, 1732.10, $1594.57,1498.13,1470.54,1266.47,1213.00,1192.61$, $1068.93,1018.40,843.32 \mathrm{~cm}^{-1}$.

\subsection{2目标化合物 2 的合成}

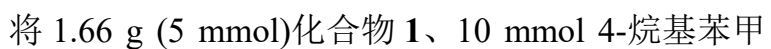
酸、 $12 \mathrm{mmol}$ 二环己基碳二亚胺(DCC)、1.2 mmol 4-二 甲氨基吡啶(DMAP)和 $50 \mathrm{~mL} \mathrm{CH} \mathrm{Cl}_{2}$ 置于 $100 \mathrm{~mL}$ 干燥 的三口烧瓶中, 室温磁力摚拌 $24 \mathrm{~h}$ (TLC 跟踪反应). 抽 滤, $\mathrm{CH}_{2} \mathrm{Cl}_{2}$ 洗涤, 减压蒸馏除去溶剂, 硅胶柱层析(淋洗 剂: 石油醚/乙酸乙酯, $V: V=30: 1)$ 分离提纯, 得目标 产物 2.

2-甲基-1,3-双(4-((4-戊基苯基)酯基)-1-( $E$ )-偶氮苯 基)苯(2a)：黄色针状固体 $2.52 \mathrm{~g}$, 收率 75.8\%. m.p. $143 \sim 144{ }^{\circ} \mathrm{C} ;{ }^{1} \mathrm{H} \mathrm{NMR}\left(400 \mathrm{MHz}, \mathrm{CDCl}_{3}\right) \delta: 8.17$ (d, $J=8$
$\mathrm{Hz}, 4 \mathrm{H}), 8.09$ (d, $J=8 \mathrm{~Hz}, 4 \mathrm{H}), 7.79(\mathrm{~d}, J=8 \mathrm{~Hz}, 2 \mathrm{H})$, 7.43 (d, $J=4.8 \mathrm{~Hz}, 4 \mathrm{H}), 7.29$ (d, $J=8 \mathrm{~Hz}, 4 \mathrm{H}), 3.15$ (s, $3 \mathrm{H}), 2.75(\mathrm{t}, J=7.6 \mathrm{~Hz}, 4 \mathrm{H}), 1.65 \sim 1.72(\mathrm{~m}, 4 \mathrm{H}), 1.33 \sim$ $1.35(\mathrm{~m}, 8 \mathrm{H}), 0.95(\mathrm{t}, J=5.6 \mathrm{~Hz}, 6 \mathrm{H}) ;{ }^{13} \mathrm{C}$ NMR $(100$ $\left.\mathrm{MHz}, \mathrm{CDCl}_{3}\right) \delta: 164.93,153.39,153.34,150.14,149.72$, $130.37,129.70,128.77,126.64 ; 125.44,124.37,122.53$, $116.50,36.15,31.94,31.17,22.73$, 14.17, 11.23; FT-IR (KBr) v: 2930.13, 2851.70, 1680.54, 1643.95, 1613.23, $1546.77,1507.93,1447.28,1354.67,1337.28,1276.20$, 1236.88, 1128.86, $1078.87 \mathrm{~cm}^{-1}$; MS $\mathrm{m} / \mathrm{z}(\%): 681.34$ $(100, \mathrm{M}+\mathrm{H})^{+}, 641.72$ (13.46), 495.57 (22.31). Anal. calcd for $\mathrm{C}_{43} \mathrm{H}_{44} \mathrm{O}_{4} \mathrm{~N}_{4}$ : C 75.86, H 6.51, N 8.23; found C 75.82, H 6.49, N 8.26.

2-甲基-1,3-双(4-((4-已基苯基)酯基)-1-(E)-偶氮苯 基)苯(2b)：黄色针状固体 $2.45 \mathrm{~g}$, 收率 $72.3 \%$. m.p. 139 $142{ }^{\circ} \mathrm{C} ;{ }^{1} \mathrm{H}$ NMR $\left(400 \mathrm{MHz}, \mathrm{CDCl}_{3}\right) \delta: 8.17$ (d, $J=$ $8 \mathrm{~Hz}, 4 \mathrm{H}), 8.10$ (d, $J=8 \mathrm{~Hz}, 4 \mathrm{H}), 7.79(\mathrm{~d}, J=8 \mathrm{~Hz}, 2 \mathrm{H})$, $7.43(\mathrm{~d}, J=8.8 \mathrm{~Hz}, 4 \mathrm{H}), 7.38(\mathrm{~d}, J=7.9 \mathrm{~Hz}, 4 \mathrm{H}), 3.15$ (s, $3 \mathrm{H}), 2.75(\mathrm{t}, J=7.6 \mathrm{~Hz}, 4 \mathrm{H}), 1.64 \sim 1.72(\mathrm{~m}, 4 \mathrm{H}), 1.33 \sim$ $1.36(\mathrm{~m}, 12 \mathrm{H}), 0.95(\mathrm{t}, J=5.6 \mathrm{~Hz}, 6 \mathrm{H}) ;{ }^{13} \mathrm{C}$ NMR $(100$ $\left.\mathrm{MHz}, \mathrm{CDCl}_{3}\right) \delta: 164.93,153.39,153.34,150.14,149.72$, $130.36,129.70,128.77,126.64,125.44,124.38,122.53$, $116.50,36.15,31.94,31.17,29.33,22.73,14.17,11.03$; FT-IR (KBr) $v: 2952.11,2841.50,1675.36,1623.45$, $1623.13,1556.78,1517.83,1457.24,1355.69,1327.18$, 1256.10, 1246.82, 1158.86, $998.87 \quad \mathrm{~cm}^{-1}$; MS $m / z(\%)$ : $709.37(100, \mathrm{M}+\mathrm{H})^{+}, 655.75$ (14.86), 523.6 (26.08). Anal. calcd for $\mathrm{C}_{45} \mathrm{H}_{48} \mathrm{O}_{4} \mathrm{~N}_{4}$ : C 76.24, H 6.83, N 7.90; found C 76.25, H 6.81, N 7.89 .

2-甲基-1,3-双(4-((4-庚基苯基)酯基)-1-(E)-偶氮苯 基)苯(2c)：黄色针状固体 $2.48 \mathrm{~g}$, 收率 71.5\%. m.p. $126 \sim 128{ }^{\circ} \mathrm{C} ;{ }^{1} \mathrm{H}$ NMR $\left(400 \mathrm{MHz}, \mathrm{CDCl}_{3}\right) \delta: 8.18(\mathrm{~d}, J=$ $8 \mathrm{~Hz}, 4 \mathrm{H}), 8.09$ (d, $J=8 \mathrm{~Hz}, 4 \mathrm{H}), 7.80$ (d, $J=8 \mathrm{~Hz}, 2 \mathrm{H})$, 7.42 (d, $J=8.4 \mathrm{~Hz}, 4 \mathrm{H}), 7.38$ (d, $J=7.2 \mathrm{~Hz}, 4 \mathrm{H}), 3.16$ (s, $3 \mathrm{H}), 2.75(\mathrm{t}, J=7.4 \mathrm{~Hz}, 4 \mathrm{H}), 1.66 \sim 1.70(\mathrm{~m}, 4 \mathrm{H}), 1.33 \sim$ $1.38(\mathrm{~m}, 16 \mathrm{H}), 0.94(\mathrm{t}, J=6.4 \mathrm{~Hz}, 6 \mathrm{H}) ;{ }^{13} \mathrm{C}$ NMR $(100$ $\left.\mathrm{MHz}, \mathrm{CDCl}_{3}\right) \delta: 164.98,152.20,151.73,150.56,149.72$, $138.42,130.38,128.79,126.66,124.42,122.54,117.83$, $36.16,31.84,31.84,31.20,29.19,22.71,14.17,11.65$; FT-IR (KBr) v: 2918.17，2849.16， 1697.63，1645.76, $1613.79,1542.68,1507.81,1450.59,1355.50,1339.12$, $1269.69,1233.43,1125.32,841.01 \mathrm{~cm}^{-1}$; MS $\mathrm{m} / z$ (\%): $737.40(100, \mathrm{M}+\mathrm{H})^{+}, 618.26$ (16.04), 630.30 (14.41), 618.27 (16.04), 651.23 (11.57). Anal. calcd for $\mathrm{C}_{47} \mathrm{H}_{52}$ $\mathrm{O}_{4} \mathrm{~N}_{4}$ : C 76.60, H 7.11, N 7.60; found C 76.56, H 7.08, N 
7.62.

2-甲基-1,3-双(4-((4-辛基苯基)酯基)-1-( $E$ )-偶氮苯 基)苯(2d): 黄色针状固体 $2.66 \mathrm{~g}$, 收率 75.2\%. m.p. $135 \sim 136{ }^{\circ} \mathrm{C} ;{ }^{1} \mathrm{H}$ NMR $\left(400 \mathrm{MHz}, \mathrm{CDCl}_{3}\right) \delta: 8.17(\mathrm{~d}, J=$ $8 \mathrm{~Hz}, 4 \mathrm{H}), 8.09$ (d, $J=8.8 \mathrm{~Hz}, 4 \mathrm{H}), 7.79$ (d, $J=8 \mathrm{~Hz}, 2 \mathrm{H}$ ), $7.43(\mathrm{~d}, J=8.8 \mathrm{~Hz}, 4 \mathrm{H}), 7.38(\mathrm{~d}, J=7.6 \mathrm{~Hz}, 4 \mathrm{H}), 3.15$ (s, $3 \mathrm{H}), 2.74(\mathrm{t}, J=7.8 \mathrm{~Hz}, 4 \mathrm{H}), 1.64 \sim 1.72(\mathrm{~m}, 4 \mathrm{H}), 1.32 \sim$ $1.37(\mathrm{~m}, 20 \mathrm{H}), 0.95(\mathrm{t}, J=6.8 \mathrm{~Hz}, 6 \mathrm{H}) ;{ }^{13} \mathrm{C}$ NMR $(100$ $\left.\mathrm{MHz}, \mathrm{CDCl}_{3}\right) \delta: 164.76,153.26,153.45,150.32,149.90$, $130.43,129.88,128.12,126.54,125.11,124.34,122.44$, $116.55,36.23,31.99,31.12,30.86,30.26,29.29,22.53$, 14.15, 11.33; FT-IR (KBr) v: 2928.13, 2841.70, 1669.54, $1632.95,1615.23,1532.77,1511.93,1454.28,1348.67$, $1342.28,1286.20,1242.88,1138.86,886.25 \mathrm{~cm}^{-1}$; MS m/z (\%): $765.43(100, \mathrm{M}+\mathrm{H})^{+}$. Anal. calcd for $\mathrm{C}_{49} \mathrm{H}_{56} \mathrm{O}_{4} \mathrm{~N}_{4}$ : C 76.93, H 7.38, N 7.32; found C 76.91, H 7.36, N 7.28.

2-甲基-1,3-双(4-((4-壬基苯基)酯基)-1-( $E$ )-偶氮苯 基)苯(2e): 黄色针状固体 $2.55 \mathrm{~g}$, 收率 70.8\%. m.p. 110 $112{ }^{\circ} \mathrm{C} ;{ }^{1} \mathrm{H}$ NMR $\left(400 \mathrm{MHz}, \mathrm{CDCl}_{3}\right) \delta: 8.17(\mathrm{~d}, J=8 \mathrm{~Hz}$, 4H), 8.09 (d, $J=8.8 \mathrm{~Hz}, 4 \mathrm{H}), 7.79$ (d, $J=8 \mathrm{~Hz}, 2 \mathrm{H}), 7.38$ (d, $J=8.8 \mathrm{~Hz}, 4 \mathrm{H}), 7.29$ (d, $J=7.6 \mathrm{~Hz}, 4 \mathrm{H}), 3.16$ (s, 3H), $2.70(\mathrm{t}, J=7.4 \mathrm{~Hz}, 4 \mathrm{H}), 1.60 \sim 1.66(\mathrm{~m}, 4 \mathrm{H}), 1.30 \sim 1.32$ (m, 24H), $0.90(\mathrm{t}, J=6.4 \mathrm{~Hz}, 6 \mathrm{H}) ;{ }^{13} \mathrm{C}$ NMR $(100 \mathrm{MHz}$, $\left.\mathrm{CDCl}_{3}\right) \delta: 164.829,153.348,153.522,150.322,149.889$, $130.325,129.652,128.553,126.650,125.322,124.427$, $122.551,116.441,36.259,31.335,31.152,30.845,30.255$, 30.015, 29.255, 22.746, 14.131, 11.241; FT-IR (KBr) v: 2920.13, 2861.70, 1666.54, 1648.97, 1622.32, 1536.67, $1517.83,1457.32,1344.57,1347.18,1266.10,1246.78$, 1130.46, $959.21 \mathrm{~cm}^{-1}$; MS m/z (\%): $793.46(100, \mathrm{M}+\mathrm{H})^{+}$, 674.32 (14.91), 686.36 (13.39), 674.33 (14.91), 679.24 (14.29). Anal. calcd for $\mathrm{C}_{51} \mathrm{H}_{60} \mathrm{O}_{4} \mathrm{~N}_{4}$ : C 77.24, H 7.63, N 7.06; found C 77.21, H 7.59, N 7.07 .

2-甲基-1,3-双(4-((4-癸基苯基)酯基)-1-(E)-偶氮苯 基)苯(2f): 黄色针状固体 $2.57 \mathrm{~g}$, 收率 69.8\%. m.p. 130 $132{ }^{\circ} \mathrm{C} ;{ }^{1} \mathrm{H}$ NMR $\left(400 \mathrm{MHz}, \mathrm{CDCl}_{3}\right) \delta: 8.13(\mathrm{~d}, J=7.6$ $\mathrm{Hz}, 4 \mathrm{H}), 8.05$ (d, $J=8.8 \mathrm{~Hz}, 4 \mathrm{H}), 7.68$ (d, $J=8 \mathrm{~Hz}, 2 \mathrm{H})$, 7.39 (d, $J=8.8 \mathrm{~Hz}, 4 \mathrm{H}), 7.35$ (d, $J=8 \mathrm{~Hz}, 4 \mathrm{H}), 3.16$ (s, $3 \mathrm{H}), 2.71(\mathrm{t}, J=7.4 \mathrm{~Hz}, 4 \mathrm{H}), 1.59 \sim 1.66(\mathrm{~m}, 4 \mathrm{H}), 1.27 \sim$ $1.32(\mathrm{~m}, 28 \mathrm{H}), 0.89(\mathrm{t}, J=6.4 \mathrm{~Hz}, 6 \mathrm{H}) ;{ }^{13} \mathrm{C}$ NMR $(100$ $\left.\mathrm{MHz}, \mathrm{CDCl}_{3}\right) \delta$ : 164.939, 153.378, 153.332, 150.132, $149.729,129.722,128.783,126.650,125.421,124.363$, 122.542, 116.456, 36.222, 31.895, 31.212, 30.758, 30.689, $30.423,30.235,29.255,22.756,14.161,11.235$; FT-IR
(KBr) v: 2920.01, 2850.68, 1728.64, 1608.83, 15993.34, $1496.65,1466.78,1416.72,1266.35,1209.43,1174.94$, 1145.96, 1066.17, $1017.92 \mathrm{~cm}^{-1}$; MS $\mathrm{m} / \mathrm{z}(\%): 821.50$ $(100, \mathrm{M}+\mathrm{H})^{+}, 702.36$ (14.40), 714.4 (12.93), 688.34 (16.11), 707.28 (13.80). Anal. calcd for $\mathrm{C}_{53} \mathrm{H}_{64} \mathrm{O}_{4} \mathrm{~N}_{4}$ : C 77.53, H 7.86, N 6.82; found C 77.51, H 7.84, N 6.79.

2-甲基-1,3-双(4-((4-十二烷基苯基)酯基)-1-(E)-偶 氮苯基)苯 $(\mathbf{2 g})$ : 黄色针状固体 $2.72 \mathrm{~g}$, 收率 71.2\%. m.p. $118 \sim 120{ }^{\circ} \mathrm{C} ;{ }^{1} \mathrm{H}$ NMR $\left(400 \mathrm{MHz}, \mathrm{CDCl}_{3}\right) \delta: 8.19$ (d, $J=$ $8 \mathrm{~Hz}, 4 \mathrm{H}), 8.11(\mathrm{~d}, J=8 \mathrm{~Hz}, 4 \mathrm{H}), 7.81(\mathrm{~d}, J=8 \mathrm{~Hz}, 2 \mathrm{H})$, 7.45 (d, $J=8.4 \mathrm{~Hz}, 4 \mathrm{H}), 7.37$ (d, $J=7.2 \mathrm{~Hz}, 4 \mathrm{H}), 3.15$ (s, $3 \mathrm{H}), 2.75(\mathrm{t}, J=7.2 \mathrm{~Hz}, 4 \mathrm{H}), 1.66 \sim 1.70(\mathrm{~m}, 4 \mathrm{H}), 1.31 \sim$ $1.37(\mathrm{~m}, 36 \mathrm{H}), 0.93(\mathrm{t}, J=6.4 \mathrm{~Hz}, 6 \mathrm{H}) ;{ }^{13} \mathrm{C}$ NMR $(100$ $\left.\mathrm{MHz}, \mathrm{CDCl}_{3}\right) \delta: 164.929,153.388,153.342,150.142$, $149.719,130.365,129.702,128.773,126.640,125.442$, $124.367,122.531,116.501,36.149,31.935,31.172$, $30.565,30.322,30.125,30.022,29.865,29.624,29.285$, 22.726, 14.171, 11.025; FT-IR (KBr) v: 2912.13, 2821.70, $1750.54,1523.95,1655.23,1506.77,1544.93,1420.28$, $1254.67,1237.28,1176.20,1136.88,1028.86,1001.87$ $\mathrm{cm}^{-1}$; MS m/z (\%): $887.56(100, \mathrm{M}+\mathrm{H})^{+}, 662.32$ (25.29), 754.40 (14.91), 745.28 (15.94). Anal. calcd for $\mathrm{C}_{57} \mathrm{H}_{72} \mathrm{O}_{4}$ $\mathrm{N}_{4}$ : C 78.04, H 8.27, N 6.39; found C 78.01, H 8.23, N 6.36 .

\section{3 化合物 $2 \mathrm{c}$ 在溶剂中的 UV 光致异构性能的测试}

化合物 $2 \mathrm{c}$ 的紫外可见吸收光谱测试使用的是 UV-8000s 紫外分光光度计. 即将目标化合物 $\mathbf{2 c}$ 溶解在 分析纯乙酸乙酯溶剂中, 配成浓度为 $1 \times 10^{-5} \mathrm{~mol} / \mathrm{L}$ 的 稀溶液, 选用石英比色典, 乙酸乙酯 (ECA, 分析纯)溶 剂作为参比液, 采用光强为 $1 \mathrm{~mW} / \mathrm{cm}^{2}$ 、波长为 $365 \mathrm{~nm}$ 的紫外光进行照射, 并对其光致异构行为进行了表征. 即将装有液晶样品溶液和空白参比液的比色㿼放在紫 外箱中, 以 $365 \mathrm{~nm}$ 的紫外波长分别照射 2, 5, 10, 30, 60 $\mathrm{s}$, 测 UV 光谱和吸光度值, 采用 $200 \sim 550 \mathrm{~nm}$ 波长扫描, 扫描速率为 $1 \mathrm{~nm} / \mathrm{s}$ (间距 $p$ 为 $1 \mathrm{~nm}$, 滤波 $S$ 为 10 ), 基线 校正后扣除空白参比液的吸收影响, 观察吸光度值, 直 至最后两次测定的吸光度值基本一样为止, 记录实验数 据.

\subsection{UV 光致异构对液晶近晶相态 $(\mathrm{Sm})$ 的影响测试}

将目标化合物 $2 \mathrm{c}$ 温度控制在 $120{ }^{\circ} \mathrm{C}$ 左右, 采用光 照强度为 $1 \mathrm{~mW} / \mathrm{cm}^{2}$ 、波长为 $365 \mathrm{~nm}$ 的紫外(UV)光对样 品照射, 通过 POM 观察 UV 照射时间与撤走 UV 光照后 时间对液晶相态性能的影响. 


\section{5 化合物 $2 \mathrm{c}$ 掺加在向列相液晶中的光致异构性能测}

试

将质量分数为 $5 \%$ 的弯曲液晶化合物 $2 \mathrm{c}$ 掺杂到母体 向列相非偶氮型棒状混合液晶材料 HAG54800-200, 其 相态为 $\left.\mathrm{Cr}-40{ }^{\circ} \mathrm{C} \mathrm{N} 99{ }^{\circ} \mathrm{C} \mathrm{I}\right)$ 中, 混合配制得到清亮 点为 $82{ }^{\circ} \mathrm{C}$ 的混合向列相液晶材料体系. 采用强度为 1 $\mathrm{mW} / \mathrm{cm}^{2}$ 的 $365 \mathrm{~nm}$ 紫外光 $(U V)$, 在 $60{ }^{\circ} \mathrm{C}$ 的温度下对该 掺杂后的混合液晶材料以及未掺杂前的母体液晶分别 进行照射, 通过 POM 观察 UV 照射时间与撤走 UV 光照 后的时间与液晶相态的变化情况的关系.

辅助材料(Supporting Information) 化合物 $\mathbf{2 a} \sim \mathbf{2 g}$ 红 外、 ${ }^{1} \mathrm{H}$ NMR、 ${ }^{13} \mathrm{C}$ NMR 谱图以及 DSC 测量结果. 这些 材料可以免费从本刊网站(http://sioc-journal.cn/)上下载.

\section{References}

[1] Rahman, M. L.; Asik, J.; Kumar, S. Liq. Cryst. 2008, 35, 1263.

[2] Bobrovsky, A.; Shibaev, V.; Hamplová, V. J. Photochem. Photobiol. A: Chem. 2016, 316, 75 .

[3] Rahman, M. L.; Asik, J.; Kumar, S.; Tschierske, C. Liq. Cryst.
2008, 35, 1263 .

[4] Nagaveni, N. G.; Roy, A.; Prasad V. J. Mater. Chem. 2012, 22, 8948.

[5] Dong, Y. M.S. Thesis, East China University of Science and Technology, Shanghai, 2010 (in Chinese). (董寅, 硕士论文, 华东理工大学, 上海, 2012.)

[6] Zhou, Y. M.S. Thesis, South China University of Science and Technology, Guangzhou, 2014 (in Chinese). (周瑛，硕士论文，华南理工大学，广州, 2014.)

[7] Rahman, M. L.; Hegde, G.; Yusoff, M. M. New J. Chem. 2013, 37, 2460.

[8] Rahman, M. L.; Sarkar, S. M.; Yusoff, M. M. $R S C A d v .2015,5$, 87019.

[9] Aralapura, Y. R.; Rahman, M. L.; Yusoff, M. M. Micro Nano Lett 2017, 12, 201.

[10] He, W. L.; Li, M.; Liu, S. Q. Liq. Cryst. 2017, 1.

[11] Duan, J.; Ma, J.; Wu, B. J. Mater. Chem. C 2014, 2, 2375.

[12] John, A. A.; Ramil, C. P.; Tian, Y. Org. Lett. 2015, 17, 6258.

[13] Sharma, S.; Han, S. H.; Han, S. Org. Lett. 2015, 17, 2852.

[14] Ding, L.; Xu, M.; Wang, J. Polymer 2014, 55, 1681.

[15] Mukhopadhyay, R. D.; Praveen, V. K.; Ajayaghosh, A. Mater. Horiz. 2014, 1, 572.

[16] Wang, W.; Wang, M. Polym. Bull. 2007, 59, 537.

[17] Zheng, H. Y.; Wei, Y. S.; Geng, W. Chem. J. Chin. Univ. 2015, 36, 899 (in Chinese)

(郑敏燕，魏永生，耿薇，高等学校化学学报, 2015, 36, 899.)

[18] Ya, Q.; Dong, X.; Chen, W. Dyes Pigm. 2008, 7, 159. 